\title{
Eating Quickly is Associated with Ultrasound-Diagnosed Non-Alcoholic Fatty Liver Disease in Japanese Adults
}

\author{
Koichiro Irie ${ }^{1}$, Tetsuji Azuma ${ }^{2}$, Takatoshi Yonenaga ${ }^{2}$, Minako Hada ${ }^{2}$, \\ Kazutoshi Watanabe ${ }^{3}$, Fumiko Deguchi ${ }^{3}$, Akihiro Obora ${ }^{3}$, Takao \\ Kojima $^{3}$ and Takaaki Tomofuji ${ }^{2 *}$ \\ ${ }^{1}$ Department of Oral Health and Preventive Dentistry, Meikai University School of \\ Dentistry, Japan \\ ${ }^{2}$ Department of Community Oral Health, School of Dentistry, Asahi University, Japan \\ ${ }^{3}$ Asahi University Hospital, Japan \\ *Corresponding Author: Takaaki Tomofuji, Department of Community Oral \\ Health, School of Dentistry, Asahi University, Mizuho, Japan.
}

Received: October 19, 2020

Published: November 18, 2020

(C) All rights are reserved by Takaaki

Tomofuji., et al.

\begin{abstract}
Non-alcoholic fatty liver disease (NAFLD) is a major global health problem. The aim of the present cross-sectional study was to investigate the relationship between eating quickly and NAFLD in Japanese adults. A total of 8556 participants underwent a health checkup. Abdominal ultrasonography was applied to diagnose NAFLD, and information on eating speed was obtained using a self-administrated questionnaire. Of the study participants, 2570 (30.0\%) had ultrasound diagnosed NAFLD. The participants who reported eating quickly had a higher prevalence of NAFLD when compared to those who did not report eating quickly. The presence of NAFLD was significantly associated with male sex (odds ratio $[\mathrm{OR}]=3.163,95 \%$ confidence interval $[\mathrm{CI}]=2.800-3.573$ ), age $\geq 65$ years $(\mathrm{OR}$ $=1.426,95 \% \mathrm{CI}=1.076-1.889)$, high body mass index $\left(\geq 25 \mathrm{~kg} / \mathrm{m}^{2} ; \mathrm{OR}=9.615,95 \% \mathrm{CI}=8.511-10.863\right)$, hypertension $(\mathrm{OR}=1.659$, $95 \% \mathrm{CI}=1.424-1.931)$, high hemoglobin A1c values $(\geq 6.5 \%$; OR $=4.096,95 \% \mathrm{CI}=3.080-5.446)$, the presence of regular exercise habits $(\mathrm{OR}=0.605,95 \% \mathrm{CI}=0.522-0.701)$, and eating quickly $(\mathrm{OR}=1.146,95 \% \mathrm{CI}=1.023-1.283)$ after adjusting for confounding factors. These results indicated that lifestyle factors, including eating quickly, is positively associated with NAFLD in Japanese adults independently of overweight and obesity.
\end{abstract}

Keywords: Eating Behaviors; Eating Speed; Fatty Liver; Cross-Sectional Study; Japanese

\section{Abbreviations}

BMI: Body Mass Index; DBP; Diastolic Blood Pressure; HbAlc: Hemoglobin A1c; NAFLD: Non-Alcoholic Fatty Liver Disease; SBP: Systolic Blood Pressure

\section{Introduction}

Non-alcoholic fatty liver disease (NAFLD) is the most common liver disease and a major global health threat due to its growing incidence and prevalence [1]. NAFLD is defined as the accumulation of lipids, mainly triacylglycerol, in the hepatocytes of individuals who do not consume significant amounts of alcohol and in whom other known causes of steatosis, such as certain chronic liver diseases (hepatitis A, B, and C) or medications, have been excluded $[2,3]$. The prevalence of NAFLD in Japanese adults is approximately $20 \%$ to $30 \%$ [4]. Nevertheless, effective pharmacological treatments for NAFLD have not been established in Japan. Therefore, investigations on various approaches to prevent and/or improve NAFLD are continuing.

Previous studies have demonstrated that lifestyle factors are closely associated with NAFLD [5-8]. For instance, it has been reported that a poor dietary composition [9] and Western dietary 
pattern [10] are important factors in the progression of NAFLD. It is also known that physical exercise is related to improvements of NAFLD [7]. However, the relationship between lifestyle and NAFLD is still not completely understood, and additional clinical studies are needed. In particular, literature on the relationship between eating behaviors and NAFLD is sparse.

Eating quickly is an inadequate eating behavior, and it has been revealed as a risk factor for overweight and obesity [11-15]. Because obesity is a cause of NAFLD [6], eating quickly may also be associated with NAFLD. However, the relationship between eating quickly and NAFLD remains unclear. We hypothesized that eating quickly is correlated to NAFLD.

\section{Purpose of the Study}

The purpose of the present cross-sectional study was to investigate the relationship between eating speed and NAFLD in Japanese adults.

\section{Materials and Methods}

Study design

The present study was conducted in accordance with the Declaration of Helsinki, and the study protocol was approved by the Ethics Committee of Asahi University (No. 27010). A total of 13778 Japanese adults, aged 29 to 82 years, who received health checkups at Asahi University Hospital in Gifu, Japan, were recruited from April 2018 to March 2019. Since the present study involved completing a survey, we did not calculate the required sample size for statistical significance. We excluded 912 participants with insufficient data. Participants who tested positive for serum antigen and antibody to hepatitis B or C infection ( $\mathrm{n}=115)$ were also excluded. In addition, we excluded participants with an alcohol intake of " $\geq$ 2 go", where "go" is a traditional Japanese unit of volume measurement corresponding to $23 \mathrm{~g}$ of ethanol ( $\mathrm{n}=1244)$. Accordingly, 8556 participants (4756 men and 3800 women) were eligible for inclusion in this study. All participants provided written informed consent prior to enrollment in the study.

\section{Diagnosis of NAFLD}

Three specialists in internal medicine diagnosed NAFLD using ultrasonography (ProSound Alpha 7, Hitachi Aloka Medical, Tokyo, Japan) [16]. An ultrasonographical diagnosis of fatty liver was defined as a bright liver, increased liver echotexture when compared to the kidneys, vascular blurring, and deep attenuation of the liver.

\section{Evaluation of eating speed and other lifestyle factors}

Information on eating speed was collected using a self-administered questionnaire, which was developed based on the con- ventional National Health and Nutrition Examination Survey and questions specified by the Industrial Safety and Health Act [17]. The participants answered "slowly", "normally", or "quickly" to the question "Compared to other people, how fast is your rate of eating?". The questionnaire also included other lifestyle factors, such as current smoking status (yes or no), regular exercise habits (absence or presence), skipping breakfast $(<3$ times/week or $\geq 3$ times/week), having dinner within 2 hours of bedtime $(<3$ times/ week or $\geq 3$ times/week), and eating snacks after dinner $(<3$ times/week or $\geq 3$ times/week). The validity and reliability of this questionnaire has already been confirmed, and it has been used in previous studies $[17,18]$.

\section{Measurement of biochemical markers}

Fasting venous blood samples were used to measure the serum levels of hemoglobin ( $\mathrm{Hb}$ ) A1c, triglyceride (TG), and high-density lipoprotein (HDL) cholesterol. The serum level of HbA1c was determined using a diabetes item automatic analyzer (DM-JACK, Kyowa Medex, Tokyo, Japan). A HbA1c value $\geq 6.5$ was defined as poor glycemic control. The serum levels of TG and HDL cholesterol were measured using a simultaneous multi-item automatic analyzer (Dimension Vista 1500, Siemens Healthineers Japan, Tokyo, Japan).

\section{Assessment of body composition}

An automatic height scale with a body composition meter (TBF110/TBF-210/DC-250, Tanita, Tokyo, Japan) was used to measure the height and body weight of the participants. The body mass index (BMI) was calculated as the weight in kilograms divided by the square of the height in meters. A BMI $\geq 25 \mathrm{~kg} / \mathrm{m}^{2}$ was classified as overweight or obesity [17].

\section{Measurement of blood pressure}

Systolic blood pressure (SBP) and diastolic blood pressure (DBP) were measured using an automatic blood pressure monitor (HBP-9021/HBP-9020/BP-230RV3, Omron Healthcare, Kyoto, Japan). In this study, a minimum of two readings was taken at intervals of at least 1 minute, and the average of those readings was used to represent the blood pressure. Hypertension was defined as a SBP $\geq 140 \mathrm{mmHg}$ or DBP $\geq 90 \mathrm{mmHg}$ [19].

\section{Statistical analysis}

The normality of our data was checked by the KolmogorovSmirnov test. Since none of the continuous variables were normally distributed, the variables are presented as medians and interquartile ranges. The chi-square test and the Mann-Whitney U test were used to assess the significance of differences in selected characteristics between the study participants with and without NAFLD and between the different eating-speed groups. 
Logistic regression analyses were also performed with the presence or absence of NAFLD as a dependent variable. Because the number of participants who reported eating slowly was small ( $n=277$ ), we combined the participants with a slow or normal eating speed into a "not eating quickly" group. Sex (male vs. female), age ( $<65$ years vs. $\geq 65$ years), BMI ( $<25$ vs. $\geq 25$ ), hypertension (absence vs. presence), HbA1c value ( $<6.5 \%$ vs. $\geq 6.5 \%$ ), current smoking status (no vs. yes), regular exercise habits (absence vs. presence), eating speed (not quickly vs. quickly), skipping breakfast ( $<3$ times/week vs. $\geq 3$ times/week), having dinner within 2 hours of bedtime ( $<3$ times/week vs. $\geq 3$ times/week), and eating snacks after dinner ( $<3$ times/week vs. $\geq 3$ times/week) were selected as independent variables. In the multivariable adjusted logistic regression analysis, independent variables were selected when the $\mathrm{p}$ value was $<0.05$ in the univariate model. In addition, we confirmed that there was no multicollinearity between each variable using Spearman's correlation analysis. Analyses were performed using a statistical package (IBM SPSS statistics version 25, IBM Japan, Tokyo, Japan). The reported p values were considered to be statistically significant if less than 0.05 .

\section{Results}

Characteristics of the study participants

The overall prevalence of NAFLD was 30\%. There were significant differences between the participants with and without NAFLD with respect to sex, age, BMI, SBP, DBP, HbA1c, TG, HDL cholesterol, current smoking status, regular exercise habit, eating speed, and frequency of having dinner within 2 hours of bedtime $(\mathrm{p}<0.001$; Table 1).

\section{Prevalence rate of NAFLD}

The prevalence of NAFLD in each eating-speed group is shown in table 2 . In total, $45.0 \%$ of the participants reported eating quickly. The prevalence of NAFLD was higher in the eating-quickly group than in the eating-slowly ( $<<0.05)$ and eating-normally $(p<0.05)$ groups.

Logistic regression analysis with the prevalence of NAFLD as the dependent variable

Table 3 shows the results of univariate logistic regression analysis with NAFLD as the dependent variable. The results showed that the odds ratios (OR) for having NAFLD were higher in those of male sex $(\mathrm{OR}=3.687,95 \%$ confidence interval $[\mathrm{CI}]=3.322-4.091)$ and in those with an age $\geq 65$ years $(\mathrm{OR}=1.695,95 \% \mathrm{CI}=1.346-2.135)$, $\mathrm{BMI} \geq 25 \mathrm{~kg} / \mathrm{m}^{2}$ (OR $=11.434,95 \% \mathrm{CI}=10.192-12.827$ ), hypertension, high $\mathrm{HbA1c}$ ( $\geq 6.5 \%$; OR $=7.469,95 \% \mathrm{CI}=2.418-3.109$ ), current smoking status $(\mathrm{OR}=1.555,95 \% \mathrm{CI}=1.371-1.763)$, the presence of regular exercise habits $(\mathrm{OR}=0.783,95 \% \mathrm{CI}=0.694$ 0.884 ), reported eating quickly (OR $=1.589,95 \% \mathrm{CI} 1.447-1.744)$, and a high frequency of dinner within 2 hours of bedtime (OR = $1.267,95 \% \mathrm{CI}=1.149-1.397$ ) than in the participants who did not have these factors ( $\mathrm{p}<0.001$ for all).

\begin{tabular}{|l|c|c|c|}
\hline \multirow{2}{*}{ Variables } & \multicolumn{2}{|c|}{ NAFLD } & \multirow{2}{*}{ p value } \\
\cline { 2 - 4 } & Absence (n= 5986) & Presence (n= 2570) & \\
\hline Male, $\mathrm{n}(\%)$ & $2794(46.7)$ & $1962(76.3)$ & $<0.001$ \\
\hline Age, years & $50(44,56)$ & $53(47,58)$ & $<0.001$ \\
\hline BMI, $\mathrm{kg} / \mathrm{m}^{2}$ & $21.4(19.7,23.3)$ & $25.5(23.5,27.7)$ & $<0.001$ \\
\hline Systolic blood pressure, mmHg & $117(107,127)$ & $126(117,135)$ & $<0.001$ \\
\hline Diastolic blood pressure, $\mathrm{mmHg}$ & $72(65,80)$ & $80.5(73,87)$ & $<0.001$ \\
\hline HbA1c, \% & $5.4(5.2,5.5)$ & $5.6(5.4,5.9)$ & $<0.001$ \\
\hline TG, mg/dL & $56(41,78)$ & $96(70,134)$ & $<0.001$ \\
\hline HDL-cholesterol, mg/dL & $67(56,79)$ & $51(44,60)$ & $<0.001$ \\
\hline Current smoker, $\mathrm{n}(\%)$ & $755(12.6)$ & $471(18.3)$ & $<0.001$ \\
\hline Regular exercise habits, $\mathrm{n}(\%)^{1}$ & $1217(20.3)$ & $428(16.6)$ & $<0.001$ \\
\hline${\text { Eating speed, } \mathrm{n}(\%)^{2}}^{2}$ & $2216(37.0)$ & $1241(48.3)$ & $<0.001$ \\
\hline Skipping breakfast, $\mathrm{n}(\%)^{3}$ & $615(10.3)$ & $291(11.3)$ & 0.156 \\
\hline Dinner within 2 hours of bedtime, $\mathrm{n}(\%)^{3}$ & $1795(30.0)$ & $904(35.2)$ & $<0.001$ \\
\hline Eating snacks after dinner, $\mathrm{n}(\%)^{3}$ & $8(0.1)$ & $3(0.1)$ & 1.000 \\
\hline
\end{tabular}

Table 1: Characteristics of the study participants with and without NAFLD.

Continuous variables are expressed as median and interquartile range.

For the significance test, Mann-Whitney U test was used for continuous variables, and Fisher's direct method was used for categories.

${ }^{1}$ : Number (\%) of presence; ${ }^{2}$ : Number (\%) of eating quickly; ${ }^{3}$ : Number (\%) of 3 or more times/week. Abbreviation: NAFLD: Non-Alcoholic Fatty Liver Disease; BMI: Body Mass Index; HbA1c: Hemoglobin A1c; TG: Triglyceride; HDL: High Density Lipoprotein. 


\begin{tabular}{|l|c|c|c|c|}
\hline & & \multicolumn{3}{|c|}{ Non-alcoholic fatty liver disease } \\
\hline Eating speed & Sample size (n) & Absence (n) & Presence (n) & Prevalence rate (\%) \\
\hline Slowly & 277 & 196 & 81 & 29.2 \\
\hline Normal & 2340 & 1443 & 897 & 38.3 \\
\hline Quickly & 2139 & 1155 & 984 & 46.0 \\
\hline
\end{tabular}

Table 2: Prevalence of non-alcoholic fatty liver disease among the different eating-speed groups. Abbreviation: n: Number.

\begin{tabular}{|c|c|c|c|c|}
\hline Variables & & Crude odds ratio & 95\% Confidence interval & p value \\
\hline \multirow{2}{*}{ Gender } & Female & 1 & (Reference) & \multirow{2}{*}{$<0.001$} \\
\hline & Male & 3.687 & $3.322-4.091$ & \\
\hline \multirow{2}{*}{ Age, years } & $<65$ & 1 & (Reference) & \multirow{2}{*}{$<0.001$} \\
\hline & $\geq 65$ & 1.695 & $1.346-2.135$ & \\
\hline \multirow{2}{*}{ BMI, kg/m² } & $<25$ & 1 & (Reference) & \multirow{2}{*}{$<0.001$} \\
\hline & $\geq 25$ & 11.434 & $10.192-12.827$ & \\
\hline \multirow{2}{*}{ Hypertension } & Absence & 1 & (Reference) & \multirow{2}{*}{$<0.001$} \\
\hline & Presence & 2.742 & $2.418-3.109$ & \\
\hline \multirow{2}{*}{$\mathrm{HbA1c} \%$} & $<6.5$ & 1 & (Reference) & \multirow{2}{*}{$<0.001$} \\
\hline & $\geq 6.5$ & 7.469 & $5.828-9.571$ & \\
\hline \multirow{2}{*}{ Current smoker } & No & 1 & (Reference) & \multirow{2}{*}{$<0.001$} \\
\hline & Yes & 1.555 & $1.371-1.763$ & \\
\hline \multirow{2}{*}{$\begin{array}{l}\text { Regular exercise } \\
\text { habits }\end{array}$} & Absence & 1 & (Reference) & \multirow{2}{*}{$<0.001$} \\
\hline & Presence & 0.783 & $0.694-0.884$ & \\
\hline \multirow{2}{*}{ Eating speed } & Not quickly & 1 & (Reference) & \multirow{2}{*}{$<0.001$} \\
\hline & Quickly & 1.589 & $1.447-1.744$ & \\
\hline \multirow{2}{*}{$\begin{array}{l}\text { Skipping break- } \\
\text { fast }\end{array}$} & $<3$ times/week & 1 & (Reference) & \multirow{2}{*}{0.148} \\
\hline & $\geq 3$ times/week & 1.115 & $0.962-1.293$ & \\
\hline \multirow{2}{*}{$\begin{array}{l}\text { Dinner within } 2 \\
\text { hours of bedtime }\end{array}$} & $<3$ times/week & 1 & (Reference) & \multirow{2}{*}{$<0.001$} \\
\hline & $\geq 3$ times/week & 1.267 & $1.149-1.397$ & \\
\hline \multirow{2}{*}{$\begin{array}{l}\text { Eating snacks } \\
\text { after dinner }\end{array}$} & $<3$ times/week & 1 & (Reference) & \multirow{2}{*}{0.841} \\
\hline & $\geq 3$ times/week & 0.873 & $0.231-3.294$ & \\
\hline
\end{tabular}

Table 3: Factors associated with NAFLD according to univariate logistic regression analysis.

Abbreviation: NAFLD: Non - Alcoholic Fatty Liver Disease; BMI: Body Mass Index; HbA1c: Hemoglobin A1c.

Table 4 shows the results of the multivariate adjusted logistic regression analysis with NAFLD as the dependent variable. NAFLD was significantly associated with male sex $(\mathrm{OR}=3.163,95 \% \mathrm{CI}=$ $2.800-3.573, \mathrm{p}<0.001)$, age $\geq 65$ years $(\mathrm{OR}=1.426,95 \% \mathrm{CI}=$ $1.076-1.889, \mathrm{p}<0.05)$, high BMI $\left(\geq 25 \mathrm{~kg} / \mathrm{m}^{2} ; \mathrm{OR}=9.615,95 \% \mathrm{CI}\right.$ $=8.511-10.863, \mathrm{p}<0.001)$, hypertension $(\mathrm{OR}=1.659,95 \% \mathrm{CI}=$
$1.424-1.931, \mathrm{p}<0.001)$, high $\mathrm{HbA1} \mathrm{c}(\geq 6.5 \%$; OR $=4.096,95 \% \mathrm{CI}=$ $3.080-5.446, \mathrm{p}<0.001)$, presence of regular exercise habits $(\mathrm{OR}=$ $0.605,95 \% \mathrm{CI}=0.522-0.701, \mathrm{p}<0.001)$, and eating quickly (OR = $1.146,95 \% \mathrm{CI}=1.023-1.283, \mathrm{p}<0.05$ ) after adjusting for sex, age, BMI, hypertension, HbA1c, current smoking status, regular exercise habits, eating speed and having dinner within 2 hours of bedtime. 


\begin{tabular}{|c|c|c|c|c|}
\hline Variables & & $\begin{array}{l}\text { Multivariable adjusted } \\
\text { odds ratio }^{1}\end{array}$ & $\begin{array}{l}\text { 95\% Confidence } \\
\text { interval }\end{array}$ & p value \\
\hline \multirow[t]{2}{*}{ Gender } & Female & 1 & (Reference) & \multirow[t]{2}{*}{$<0.001$} \\
\hline & Male & 3.163 & $2.800-3.573$ & \\
\hline \multirow[t]{2}{*}{ Age, years } & $<65$ & 1 & (Reference) & \multirow[t]{2}{*}{0.014} \\
\hline & $\geq 65$ & 1.426 & $1.076-1.889$ & \\
\hline \multirow[t]{2}{*}{ BMI, $\mathrm{kg} / \mathrm{m}^{2}$} & $<25$ & 1 & (Reference) & \multirow[t]{2}{*}{$<0.001$} \\
\hline & $\geq 25$ & 9.615 & $8.511-10.863$ & \\
\hline \multirow[t]{2}{*}{ Hypertension } & Absence & 1 & (Reference) & \multirow[t]{2}{*}{$<0.001$} \\
\hline & Presence & 1.659 & $1.424-1.931$ & \\
\hline \multirow[t]{2}{*}{$\mathrm{HbA1c}, \%$} & $<6.5$ & 1 & (Reference) & \multirow[t]{2}{*}{$<0.001$} \\
\hline & $\geq 6.5$ & 4.096 & $3.080-5.446$ & \\
\hline \multirow{2}{*}{$\begin{array}{l}\text { Regular exercise } \\
\text { habits }\end{array}$} & Absence & 1 & (Reference) & \multirow[t]{2}{*}{$<0.001$} \\
\hline & Presence & 0.605 & $0.522-0.701$ & \\
\hline \multirow[t]{2}{*}{ Eating speed } & Not quickly & 1 & (Reference) & \multirow[t]{2}{*}{0.018} \\
\hline & Quickly & 1.146 & $1.023-1.283$ & \\
\hline \multirow{2}{*}{$\begin{array}{l}\text { Dinner within } 2 \\
\text { hours of bedtime }\end{array}$} & $<3$ times/week & 1 & (Reference) & \multirow[t]{2}{*}{0.056} \\
\hline & $\geq 3$ times/week & 0.889 & $0.789-1.003$ & \\
\hline
\end{tabular}

Table 4: Factors associated with NAFLD according to multivariable adjusted logistic regression analysis.

${ }^{1}$ : Adjusting gender, age, BMI, hypertension, HbA1c, regular exercise habits, eating speed, and dinner within 2 hours of bedtime.

Abbreviation: NAFLD: Non-Alcoholic Fatty Liver Disease; BMI: Body Mass Index; HbA1c: Hemoglobin A1c.

\section{Discussion}

To our knowledge, this is the first study to investigate the relationship between eating quickly and NAFLD in Japanese adults. In this study, the prevalence of NAFLD was higher in the eating-quickly group than in the eating-slowly group or the eating-normally group. In addition, the logistic regression analyses showed that the presence of NAFLD was significantly correlated to eating quickly as well as to male sex, age $\geq 65$ years, high BMI, hypertension, high HbA1c, and absence of regular exercise habits after adjusting for sex, age, BMI, hypertension, HbA1c, current smoking status, regular exercise habits, eating speed, and having dinner within 2 hours of bedtime. These results indicated that there is a positive association between eating quickly and NAFLD in Japanese adults.

Eating quickly and having dinner within 2 hours of bedtime are risk factors for overweight and obesity $[20,21]$. The recent increase in the prevalence and severity of NAFLD has been linked to the rising trends in obesity [22]. Therefore, it is possible that overweight and obesity overwhelm the effects that eating quickly and having dinner within 2 hours of bedtime have on NAFLD. In our regres- sion analysis, a significant association between eating quickly and NAFLD remained even after adjusting for overweight and obesity. In contrast, there was no association between having dinner within 2 hours of bedtime and NAFLD after adjusting for overweight and obesity. These results suggested that eating quickly, but not having dinner within 2 hours of bedtime, was associated with NAFLD independently of overweight and obesity.

A possible mechanism by which eating quickly is related to NAFLD is that eating quickly might cause overeating before the stomach can sense fullness [13]. Overeating following eating quickly would lead to being overweight and obesity [15,20-22], and dysfunctional adipocytokine secretion in obese conditions contribute to the development of NAFLD [23]. In our findings, overweight or obesity is strongly associated with the presence of NAFLD. Although the positive association between eating quickly and NAFLD was statistically significant after adjusting for overweight and obesity, it is possible that eating quickly had indirect effects on the presence of NAFLD through overweight and obesity. 
Our results also showed that eating quickly was positively associated with poor glycemic control (HbA1c $\geq 6.5 \%$ ) and hypertension. Poor glycemic control and hypertension can lead to the acceleration of lipolysis in adipose tissues [24,25]. It has been suggested that poor glycemic control and hypertension accompanied by eating quickly may also affect the presence of NAFLD.

In Japan, the Japanese Government started a new health policy of providing specific health checkups followed by specific counseling for individuals diagnosed with metabolic syndrome since 2008. From this project, it was reported that the improvements of BMI and waist circumference in those with metabolic syndrome were greater among those who participated in the specific health guidance than in those who did not participate [26]. Furthermore, it was also revealed that intensive health guidance focusing on eating quickly was more effective for improving BMI, waist circumference, and triglyceride levels than the standard specific health guidance in subjects with metabolic syndrome [27]. These indicated that interventions for eating quickly may have additional effects on preventing and/or improving NAFLD following other lifestyle modifications. However, further studies are needed to clarify this point.

From our observations, eating snacks after dinner was not associated with the presence of NAFLD. Only 11 participants reported eating snacks after dinner in the present population, which may have been too few to show any statistical significance.

In this study, the prevalence of NAFLD was approximately $30 \%$. This value is similar to a previous report, which showed that the prevalence of NAFLD was $20 \%$ to $30 \%$ in the Japanese general population [4]. In addition, $40.4 \%$ of the participants in our study reported eating quickly. This value is also similar to the results of past studies, which have reported the prevalence of eating quickly to be $40 \%$ to $50 \%$ among Japanese adults [28,29].

The strength of this study is the large sample size that was sufficient for assessing the prevalence of NAFLD among participants who reported eating quickly. However, our study had some limitations. First, all participants had received health checkups at Asahi University Hospital, which may limit our ability to extrapolate the findings to the general population. Second, since the present study was combined with routine health checkups, the amount of data we could collect was limited, and the questionnaire did not include any questions regarding the total energy intake or the eating fre- quency per day. Third, this study was a cross-sectional study, which prevented conclusions to be reached regarding causal relationships. Additional longitudinal studies are needed to investigate the relationship between eating speed and NAFLD.

\section{Conclusion}

Within the limits of our study, eating quickly was positively associated with NAFLD in Japanese adults. Interventions for eating quickly may be beneficial for controlling the risk of NAFLD.

\section{Acknowledgements}

This research received no external funding.

\section{Conflict of Interest}

The authors declare no conflicts of interest.

\section{Bibliography}

1. Perdomo CM., et al. "Impact of nutritional changes on nonalcoholic fatty liver disease”. Nutrients 11.3 (2019): 677.

2. Marchesini G., et al. "Association of nonalcoholic fatty liver disease with insulin resistance". American Journal of Medicine 107.5 (1999): 450-455.

3. Marchesini G., et al. "Nonalcoholic fatty liver disease: A feature of the metabolic syndrome". Diabetes 50.8 (2001): 1844-1850.

4. Eguchi Y., et al. "Prevalence and associated metabolic factors of nonalcoholic fatty liver disease in the general population from 2009 to 2010 in Japan: a multicenter large retrospective study". Journal of Gastroenterology 47.5 (2012): 586-595.

5. Kaur J. "A comprehensive review on metabolic syndrome". Cardiology Research and Practice 2014 (2014): 943162.

6. Stefan N., et al. "Non-alcoholic fatty liver disease: Causes, diagnosis, cardiometabolic consequences, and treatment strategies". Lancet Diabetes and Endocrinology 7.4 (2019): 313-324.

7. Zou TT., et al. "Lifestyle interventions for patients with nonalcoholic fatty liver disease: a network meta-analysis". European Journal of Gastroenterology 30.7 (2018): 747-755.

8. Maria Garralda-Del-Villar., et al. "Healthy lifestyle and incidence of metabolic syndrome in the SUN cohort". Nutrients 11.1 (2019): 65.

9. Eslamparast T., et al. "Dietary composition independent of weight loss in the management of non-alcoholic fatty liver disease". Nutrients 9.8 (2017): 800. 
10. Wong VW., et al. "Beneficial effects of lifestyle intervention in non-obese patients with non-alcoholic fatty liver disease". Journal of Hepatology 69.6 (2018): 1349-1356.

11. Ohkuma T., et al. "Association between eating rate and obesity: a systematic review and meta-analysis". International Journal of Obesity 39.11 (2015): 1589-1596.

12. Carnell S and Wardle J. "Measuring behavioural susceptibility to obesity: validation of the child eating behaviour questionnaire". Appetite 48.1 (2007): 104-113.

13. Guertin TL., et al. "Eating behavior of bulimics, self-identified binge eaters, and non-eating-disordered individuals: what differentiates these populations?" Clinical Psychology Review 19.1 (1999): 1-23.

14. Otsuka R., et al. "Eating fast leads to obesity: findings based on self-administered questionnaires among middle-aged Japanese men and women". Journal of Epidemiology 16.3 (2006): 117-124.

15. Hill AJ. "Obesity and eating disorders". Obesity Reviews 8.1 (2007): 151-155.

16. Chitturi S., et al. "Non-alcoholic fatty liver disease in the Asia-pacific region: Definitions and overview of proposed guidelines". Journal of Gastroenterology and Hepatology 22.6 (2007): 778-787.

17. Iwasaki T., et al. "Self-reported behavior of eating quickly is correlated with visceral fat area in Japanese non-obese adults". Asia Pacific Journal of Clinical Nutrition 28.1 (2019): 92-98.

18. Mochizuki K., et al. "Self-reported faster eating is positively associated with accumulation of visceral fat in middle-aged apparently healthy Japanese men". European Journal of Nutrition 53.5 (2014): 1187-1194.

19. Otani. K., et al. "Prevalence and correlates of hypertension among Japanese adults, 1975 to 2010". International Journal of Environmental Research and Public Health 15.8 (2018): 1645.

20. Maruyama K., et al. "The joint impact on being overweight of self reported behaviours of eating quickly and eating until full: cross sectional survey". British Medical Journal 337 (2008): a2002.

21. Okada C., et al. "The association of having a late dinner or bedtime snack and skipping breakfast with overweight in Japanese women". Journal of Obesity (2019): 2439571.
22. Sun Y., et al. "Lifestyle and overweight among Japanese adolescents: the Toyama Birth Cohort Study". Journal of Epidemiology 19.6 (2009): 303-310.

23. Cao H. "Adipocytokines in obesity and metabolic disease". Journal of Endocrinology 220.2 (2014): T47-T59.

24. Achila 00., et al. "Factors associated with poor glycemic and lipid levels in ambulatory diabetes mellitus type 2 patients in Asmara, Eritrea: A cross-sectional study". Journal of Diabetes Research 2020 (2020): 5901569.

25. Vaněčková I., et al. "Obesity-related hypertension: possible pathophysiological mechanisms". Journal of Endocrinology 223.3 (2014): R63-R78.

26. Matsuzawa, Y. "Specific health guidance, the nationwide lifestyle intervention program targeting metabolic syndrome, seems to be successful in Japan". Journal of Atherosclerosis and Thrombosis 25.4 (2018): 304-305.

27. Ekuni D., et al. "Association between intensive health guidance focusing on eating quickly and metabolic syndrome in Japanese middle-aged citizens". Eating and Weight Disorders 25.1 (2020): 91-98.

28. Nagahama S., et al. "Self-reported eating rate and metabolic syndrome in Japanese people: cross-sectional study". BMJ Open 4.9 (2014): e005241.

29. Gouda M., et al. "Association between eating habits and glycemic control and obesity in Japanese workers with type 2 diabetes mellitus". Diabetes, Metabolic Syndrome and Obesity 11 (2018): 647-658.

\section{Assets from publication with us}

- Prompt Acknowledgement after receiving the article

- Thorough Double blinded peer review

- Rapid Publication

- Issue of Publication Certificate

- High visibility of your Published work

Website: www.actascientific.com/

Submit Article: www.actascientific.com/submission.php

Email us: editor@actascientific.com

Contact us: +919182824667 